Research Article

\title{
Synergic Involvements of Microorganisms in the Biomedical Increase of Polyphenols and Flavonoids during the Fermentation of Ginger Juice
}

\author{
Christian Aimé Kayath (D, , ${ }^{1,2}$ Armel Ibala Zamba, ${ }^{2,3}$ Saturnin Nicaise Mokémiabeka,,2 \\ Meddy Opa-Iloy, ${ }^{1,2}$ Paola Sandra Elenga Wilson,, ${ }^{1,2,3}$ Moïse Doria Kaya-Ongoto,, ${ }^{1,2}$ \\ Rodd Jurah Mouellet Maboulou, ${ }^{1,2,3}$ and Etienne Nguimbi ${ }^{1,2}$ \\ ${ }^{1}$ Laboratoire de Biologie Cellulaire et Moléculaire (BCM), Faculté des Sciences et Techniques, Université Marien Ngouabi, BP. 69, \\ Brazzaville, Congo \\ ${ }^{2}$ Institut National de Recherche en Sciences Exactes et Naturelles (IRSEN), Avenue de l'Auberge Gascogne, BP. 2400, \\ Brazzaville, Congo \\ ${ }^{3}$ Ecole Nationale Supérieure d'Agronomie et de Foresterie, Université Marien Ngouabi, BP. 69, Brazzaville, Congo
}

Correspondence should be addressed to Christian Aimé Kayath; chriskayath@yahoo.fr

Received 20 January 2020; Revised 15 May 2020; Accepted 20 July 2020; Published 1 August 2020

Academic Editor: Todd R. Callaway

Copyright ( 12020 Christian Aimé Kayath et al. This is an open access article distributed under the Creative Commons Attribution License, which permits unrestricted use, distribution, and reproduction in any medium, provided the original work is properly cited.

\begin{abstract}
Steered fermentation by microorganisms gives great added value in the nutritional quality of local food. Ginger rhizome naturally contains a myriad of bioactive compounds including polyphenol and flavonoids. The aim of this work was to ferment the ginger juice, to evaluate the biochemical parameters of ginger wine, and to understand the involvement of microorganisms in the bioincrease of polyphenol compounds. Titratable acidity and $\mathrm{pH}$ values were determined and showed that $\mathrm{pH}$ is around 1.6 at the end of the fermentation when the acidity is around $6.431 \mathrm{~g} / \mathrm{L}$. Using colorimetric assay, the total polyphenolic and flavonoid compounds were evaluated throughout the fermentation. The variation of the polyphenol and flavonoid concentrations of the unsweetened sample was around 10.18 to $14.64 \mathrm{mg}$ Eq AG/g and 1.394 to $2.224 \mathrm{mg}$ Eq Cat/g Ms, but those from the sweet sample were around 10.82 to $18.34 \mathrm{mg}$ Eq AG/g Ms and 1.311 to $2.290 \mathrm{mg}$ Eq Cat/g. Using onestep PCR, multiplex techniques with specific primers, with yeast-like phenotype $27.27 \%$ (6), have been assigned among 22 isolates to Saccharomyces cerevisiae. By using PCR multiplex techniques, Bacillus licheniformis, Bacillus pumilus, Bacillus safensis, and Saccharomyces cerevisiae have been identified. Together with Saccharomyces cerevisiae, we showed that Bacillus sp. are able to secrete enzymatic landscape with some activities up to $50 \%$ including cellulase, amylase, pectinase, and protease.
\end{abstract}

\section{Introduction}

Ginger, from its scientific name Zingiber officinale Roscoe, is a plant of the genus Zingiber and member of the family Zingiberaceae [1,2]. This plant, originally from Asia, is currently cultivated in many tropical regions [3]. The rhizome is underground stem used in traditional medicine and in cooking for the preparation of infusions, drinks, and spices. In the Republic of the Congo, ginger is cultivated and consumed in raw form (rhizome), condiments, or as a drink. Ginger juice is among the most locally commercialized juice throughout in Republic of the Congo cities. No published studies have notified that, more and more, the local populations consume a fermented drink based on ginger juice.

The crop of rhizome has been known to harbor multiple virtues such as lowering cholesterol levels and preventing cancer [4]. Ginger rhizomes naturally contain polyphenols which are a group of commonly encountered antioxidants 
[5]. These compounds of biological interest are characterized by the presence of at least one phenolic motif (aromatic cycle on which one or more -OH groups are grafted). One of the peculiarities of polyphenols lies in their incredible diversity. There are currently more than 8000 phenolic compounds, including 5000 for the subclass of flavonoids. These biomolecules have anticancer and antisclerotic properties $[6,7]$ and hepatoprotective and antiallergic effects [8,9]. Several studies have evaluated a myriad of health benefits of ginger in terms of its antidiabetic effect linked to major bioactive constituents [10]. The intracellular localization of the polyphenol compound is well known. It was previously documented that polyphenolic compounds have been found in the vacuole, and a number of them are close to the pectocellulosic wall of plant cells [11]. Many microorganisms like bacteria, fungi, and yeasts are known to secrete enzymes capable of degrading the cell wall and increasing the quantity of released phenolic compounds [12-18].

The choice of rhizome is justified by the fact that ginger juice is the traditional cold drink that is the most consumed in the Republic of the Congo, and ginger contained several bioactive compounds [1, 4-10]. To our knowledge on more cited popular databases, no study has been carried out on attempts to ferment ginger juice by showing the bioincrease of bioactive compounds. We are interested in the involvement of microorganisms in the nutrient values bioincreased during fermentation. The analysis of the microbiological and biochemical quality of this wine will be of major interest for this work.

\section{Methods}

2.1. Preparation of Fermented Ginger, Determination of $\mathrm{pH}$, Titratable Acidity, and Alcohol Content of Ginger Wine. The ginger rhizomes were first bought at Brazzaville local markets including Bacongo, Makélékéle, and Bouemba Markets. $80 \mathrm{~g}$ of ginger rhizomes was vigorously washed and ground and $1 \mathrm{~L}$ of water was added (i.e., 10\%). After sifting the mixture, $120 \mathrm{~g}$ of sucrose (Nkayi) was added (i.e., 12\%). The mixture was distributed in eight containers containing $1 \mathrm{~L}$ of the samples dedicated to be tested. The fermentation process of each sample was followed for seven days. The first day has been considered as Day 1 and so on till day 7 . Samples were systematically analyzed for biochemical and microbiological qualities. By using HANNA pH-meter HI 99161, $\mathrm{pH}$ values of the ginger wine were determined each day by direct measurement. In the side experiments, samples have been performed for the titratable acidity of the ginger wine during fermentation by titrimetry using a $0.05 \mathrm{~N}$ sodium hydroxide solution, in the presence of $1 \%$ phenolphthalein as an indicator. The calculation of the alcohol rate has been done at the end of fermentation by using seven samples of fermented ginger wine (E1, E2, E3, E4, E5, E6, and E7) at the end of the fermentation (day 7), and this was extended until the twentieth day (day 20). $250 \mathrm{~mL}$ of each fermented ginger wine samples was measured, poured into a $1 \mathrm{~L}$ flask, and then connected to the refrigerant. The fermented drink was heated to the boiling point. Distillation is stopped after collecting more than $3 / 4$ of the volume of the test tube. For this, heating is stopped, the flask is cooled to remove the remaining wine, and the distillate is then brought to room temperature of the wine. The percentage of alcohol is evaluated at $20^{\circ} \mathrm{C}$ by using an alcoholometer.

\subsection{Extraction of Phenolic Compounds}

2.2.1. Drying of Ginger Roots and Fermentation. The ginger roots were washed thoroughly with water and cut into thin rings. After having ground these rings with the aid of a mortar, the grounded product obtained was dried in an oven set at $50^{\circ} \mathrm{C}$ for 72 hours. The extracts to be used for the determination of total polyphenols were obtained by mixing $50 \mathrm{~g}$ of the dried plant material in $500 \mathrm{~mL}$ (10\%) of distilled water in Erlenmeyer flask. The mixtures are stirred for 72 hours and then filtered. The filtrate obtained is distributed in six small vials due to $30 \mathrm{~mL}$. Another part of the filtrate is chaptalized by adding $20 \mathrm{~g}$ of the sugar (SARIS, Nkayi) (4\%) until complete dissolution. The samples are analyzed at T0 (first day), T1 (4 days of fermentation), T2 (8 days of fermentation), T3 (12 days of fermentation), and T4 (16 days of fermentation). The contents of vials T0 to T4 have been kept at shelter from light waiting to be analyzed.

2.2.2. Determination of Total Polyphenols. The reagent used is the "Folin-Ciocalteu" reagent. The total polyphenolic compounds are determined in the following manner. $0.1 \mathrm{~mL}$ of the plant extract is introduced into Eppendorf tube, the extract is then diluted with $0.9 \mathrm{~mL}$ of distilled water, then $0.9 \mathrm{~mL}$ of the Folin-Ciocalteu reagent $(1 \mathrm{~N})$ is added, and then immediately, $0.2 \mathrm{~mL}$ of $\mathrm{Na}_{2} \mathrm{CO}_{3}$ solution (20\%) is added. The mixture obtained is incubated at room temperature for about 40 minutes, protected from light. The absorbance is then measured using a spectrophotometer at $725 \mathrm{~nm}$ with a methanol solution used as a blank. Calibration line is previously carried out before analysis with gallic acid under the same conditions as the samples to be analyzed. The results obtained are expressed in $\mathrm{mg}$ gallic acid equivalent per gram of dry matter (E AG/g Ms).

2.2.3. Determination of Total Flavonoids. The reagents used consist of the colorless solution of sodium nitrite $\left(\mathrm{NaNO}_{2}, 5 \%\right)$ and aluminum chloride $\left(\mathrm{AlCl}_{3}, 10 \%\right)$. The total flavonoids are evaluated by colorimetry; $250 \mu \mathrm{L}$ of the extract and $1 \mathrm{~mL}$ of distilled water are successively introduced into a $10 \mathrm{ml}$ flask. At the initial time ( 0 minutes), $75 \mu \mathrm{L}$ of a solution of $\mathrm{NaNO}_{2}(5 \%)$ is added; after 5 minutes, $75 \mu \mathrm{L}$ of $\mathrm{AlCl}_{3}(10 \%)$ is added. And after 6 minutes, $500 \mu \mathrm{L}$ of $\mathrm{NaOH}(1 \mathrm{~N})$ is added and $2.5 \mathrm{~mL}$ of distilled water is added successively to the mixture. A calibration curve is drawn up with standard solutions of catechin prepared at different concentrations. The absorbance of the mixture obtained is directly measured with a UV-visible spectrophotometer at $510 \mathrm{~nm}$, and the results are expressed in $\mathrm{mg}$ equivalent catechin/gr of dry matter (EC/g Ms).

2.3. Isolation of Microorganisms. At the end of 7 days of fermentation, the ginger wine was collected to make 
microbiology assessment. Dilutions were done, and microorganism suspension was streaked on Sabouraud supplemented with chloramphenicol, $100 \mu \mathrm{g} / \mathrm{mL}$, and Mossel supplemented with polymyxin B. Enumeration of colonies was done in triplicate on plate count agar (PCA). The Petri dishes were incubated at $37^{\circ} \mathrm{C}$ for $24 \mathrm{~h}$ to $48 \mathrm{~h}$. After the first isolation on Petri dishes, each colony of different appearance was separately isolated. Purification of the isolates was rigorously done by successive and alternating subcultures. Purity was estimated by using a microscope for morphological characterization. Gram status was determined by using 3\% KOH. Sporulation, hydrogen peroxide $\left(\mathrm{H}_{2} \mathrm{O}_{2}\right)$, and oxidases tests were used for biochemical characterization.

\subsection{Determination of Proteolytic Activity. Some Bacillus} strains were assessed for the ability to secrete proteases in the extracellular environment as described and modified by Kaya-Ongoto et al. [19]. Briefly, $1 \mathrm{~g}$ of agarose was weighed and mixed with $100 \mathrm{~mL}$ of PBS. The mixture was heated in a microwave for $3 \mathrm{~min}$ until agarose was completely dissolved and then cooled in a water bath at $40^{\circ} \mathrm{C}$. Then, $10 \mathrm{~mL}$ of skim milk was added to the mixture. After homogenization, the mixture was poured into the Petri dishes. Once solidified, wells were carefully and aseptically generated into the gels. A volume of $50 \mu \mathrm{L}$ of the overnight culture supernatant is deposited in the wells made on the agar medium composed of $1 \%$ agarose gel, $0.01 \mathrm{M}$ PBS, pH 7.4, and skimmed milk. The Petri dishes are incubated at $37^{\circ} \mathrm{C}$ for 24 hours. The presence of proteolytic activity is detected by a clear halo around colonies indicating hydrolysis of casein. The halo diameters were measured.

2.5. Amylolytic, Cellulolytic, and Pectinolytic Activities. To assess the amylolytic, cellulolytic, and pectinolytic activities, an overnight culture was carried out on Petri dishes containing LB medium for the purpose of well-isolated colonies. Then, a young bacterial colony was deposited on the surface on solid LB medium separately added to $1 \%$ of starch, $0.5 \%$ of cellulose, and $0.5 \%$ of pectin. The Petri dishes are incubated $48 \mathrm{~h}$ to $72 \mathrm{~h}$. The revelation is made with Lugol. A test is positive if there is a clear halo around the colony corresponding to a lysis range. The percentage of enzymatic activity was determined according to the following formula: $\%=$ DT $-\mathrm{DC} / \mathrm{DT}$ with DT: total lysis diameter (lysis area+ colony diameter); DC: colony diameter; and \%: percentage of lysis.

2.6. Molecular Identification of Microorganisms. The recent molecular identification using the fibE gene encoding for the fibrinolytic enzyme has been used for targeting strains like Bacillus amyloliquefaciens, B. subtilis, B. pumilus, B. licheniformis, B. altitudinis, B. mojavensis, B. safensis, and B. atrophaeus. Yeast has been identified by using the molecular methods. Primers used in this work are indicated in Table 1 with the corresponding targeted microorganisms. Extraction and purification of isolate genomic DNA were performed according to the NucleoSpin Microbial DNA (MachereyNAGEL) kit. Briefly, isolates were grown in $5 \mathrm{~mL}$ LB broth for $24 \mathrm{~h}$ at $37^{\circ} \mathrm{C}$ with stirring. The DNA purity was assessed by electrophoresis on agarose gel and by the ratio of optical densities $260 / 280 \mathrm{~nm} .5 \mu \mathrm{L}$ of each amplification product was mixed with $2 \mu \mathrm{L}$ of loading buffer (BIOKE). Mixtures were subjected to electrophoresis on $1 \%$ agarose gel $(\mathrm{w} / \mathrm{v})$. The $10 \mathrm{~kb}$ 2-Log (BIOKE) was used as a molecular weight marker.

\section{Statistical Analysis}

Principal component analysis (PCA) was used to investigate possible correlations between isolates and enzymatic activity. Prior to ordination, percentage of enzymatic activity data was transformed to better meet the assumptions of normality [21] using $\ln (x+1)$. All analyses were performed using CANOCO (Canonical Community Ordination, version 4.5) [22].

\section{Results}

4.1. Obtaining the Ginger Wine Fermentation Diagram. Ginger wine is not a local drink. We tried to make this drink by performing the chaptalization technique of adding sugar with ginger juice. Figure 1 shows us the manufacturing diagram for the lab fermentation of ginger juice.

4.2. Evaluation of Biochemical Parameters. The measurements of the $\mathrm{pH}$ values obtained from the $1^{\text {st }}$ day of fermentation until the $7^{\text {th }}$ of fermentation are presented (Figure 2). After sieving and just the first day of fermentation, $\mathrm{pH}$ of ginger is around 4.5 and decreases considerably at the end of fermentation to 1.6 (Figure 2(a)). As explained in the methods, the ginger wine was distilled after seven days; the alcohol level was around $7 \%$. To optimize the alcohol level, the fermentation was extended until the twentieth day. The results show a clear increase in the alcohol rate up to $45 \%$ (Figure 2(c)). In order to know the type of fermentation of ginger juice, we carried out titratable acidity. The values are between $1.687 \mathrm{~g} / \mathrm{L}$ and $6.421 \mathrm{~g} / \mathrm{L}$ (Figure 2(b)).

4.3. Determination of Total Polyphenols and Flavonoids. Polyphenols constitute a family of organic molecules widely present in the plant kingdom. They are characterized, as the name suggests, by the presence of at least two phenolic groups associated in more or less complex structures, generally of high molecular weight. In order to quantify these molecules, we carried out as mentioned in the methods. As results, we showed that for the unsweetened sample, the polyphenol concentration varies from 10.18 to $14.9 \mathrm{mg} \mathrm{Eq} \mathrm{AG/g} \mathrm{Ms} \mathrm{after} 16$ days of fermentation (Figure 3(a)). As for the sweet sample, the polyphenol concentration ranges from 10.82 to $18.3 \mathrm{mg} \mathrm{Eq} \mathrm{AG/g} \mathrm{Ms}$ after 20 days of fermentation (Figure 3(a)). It can be seen that the polyphenol concentrations in the sweet sample are higher than those in the unsweetened sample (Figure 3). For 
Table 1: Primers used in this work.

\begin{tabular}{|c|c|c|c|c|}
\hline Oligo names & $5^{\prime}----3^{\prime}$ & Size $(\mathrm{pb})$ & Targeted species & References \\
\hline \multicolumn{5}{|c|}{ For group 1 Bacillus identification } \\
\hline Ba.IdMa-F & GCGCAGTCCGTGCCTTACGGCGT & \multirow{2}{*}{828} & \multirow{2}{*}{ B. amyloliquefaciens } & \multirow{16}{*}[19]{} \\
\hline Ba.IdMa-R & TTACTGAGCTGCCGCCTGTACG & & & \\
\hline Bl.Id.Ma-F & GCGCAAACCGTTCCTTACGGCAT & \multirow{2}{*}{825} & \multirow{2}{*}{ B. licheniformis } & \\
\hline Bl.Id.Ma-R & TTATTGAGCGGCAGCTTCGAC & & & \\
\hline Bs.Id.Ma-F & GCGCAATCTGTTCCTTATGGCAT & \multirow{2}{*}{835} & \multirow{2}{*}{ B. subtilis } & \\
\hline Bs.Id.Ma-R & TTATTGTGCAGCTGCTTGTACGTTGA & & & \\
\hline Bp.Id.Ma-F & GCACAAACCGTCCCTTATGGAAT & \multirow{2}{*}{828} & \multirow{2}{*}{ B. pumilus } & \\
\hline Bp.Id.Ma-R & TTAGTTAGAAGCCGCTTGAGCG & & & \\
\hline Bm.Id.Ma-F & GCGCAATCTGTTCCTTACGGCAT & \multirow[b]{2}{*}{837} & \multirow[b]{2}{*}{ B. mojavensis } & \\
\hline Bm.Id.Ma-R & TTATTGTGCAGCTGCCTGCAC & & & \\
\hline Bsa.Id.Ma-F & GCACAAACCGTCCCTTATGGAAT & \multirow{2}{*}{828} & \multirow{2}{*}{ B. safensis } & \\
\hline Bsa.Id.Ma-R & TTAGTTAGAAGCCGCTTGAACGTTG & & & \\
\hline Bat.Id.Ma-F & GCTCAGTCA GTACCTTATG GCAT & \multirow[b]{2}{*}{828} & \multirow[b]{2}{*}{ B. atrophaeus } & \\
\hline Bat.Id.Ma-R & TTATTGCGCTGCTGCCTGAACG & & & \\
\hline Bal.Id.Ma-F & GGTCAAAGCGTCCCTTATGGTA & \multirow{2}{*}{828} & \multirow{2}{*}{ B. altitudinis } & \\
\hline Bal.Id.Ma-R & TTATCGTGCAGCTTTTTGTAC & & & \\
\hline \multicolumn{4}{|c|}{ For yeast identification } & \multirow{13}{*}[20]{} \\
\hline Sbay F1 & GCTGACTGCTGCTGCTGCCCCCG & \multirow{2}{*}{275} & \multirow[b]{2}{*}{ Saccharomyces bayanus } & \\
\hline Sbay R1 & TGTTATGAGTACTTGGTTTGTCG & & & \\
\hline Scer F2 & GCGCTTTACATTCAGATCCCGAG & \multirow{2}{*}{150} & \multirow{2}{*}{ Saccharomyces cerevisiae } & \\
\hline Scer R2 & TAAGTTGGTTGTCAGCAAGATTG & & & \\
\hline Sarb_F1 & GGCACGCCCTTACAGCAGCAA & \multirow{2}{*}{349} & \multirow{2}{*}{ Saccharomyces arboricolus } & \\
\hline Sarb_R2 & TCGTCGTACAGATGCTGGTAGGGC & & & \\
\hline Skud_F2 & ATCTATAACAAACCGCCAAGGGAG & \multirow[b]{2}{*}{660} & \multirow[b]{2}{*}{ Saccharomyces kudriavzevii } & \\
\hline Skud_R1 & CGTAACCTACCTATATGAGGGCCT & & & \\
\hline Smik_F1 & ACAAGCAATTGATTTGAGGAAAAG & \multirow{2}{*}{508} & \multirow{2}{*}{ Saccharomyces mikatae } & \\
\hline Smik_R1 & CCAGTCTTCTTTGTCAACGTTG & & & \\
\hline Spar_F7 & СTTTCTACСССТTCTCCATGTTGG & \multirow{2}{*}{739} & & \\
\hline Spar_R7 & CAATTTCAGGGCGTTGTCCAACAG & & Saccharomyces paradoxus & \\
\hline
\end{tabular}

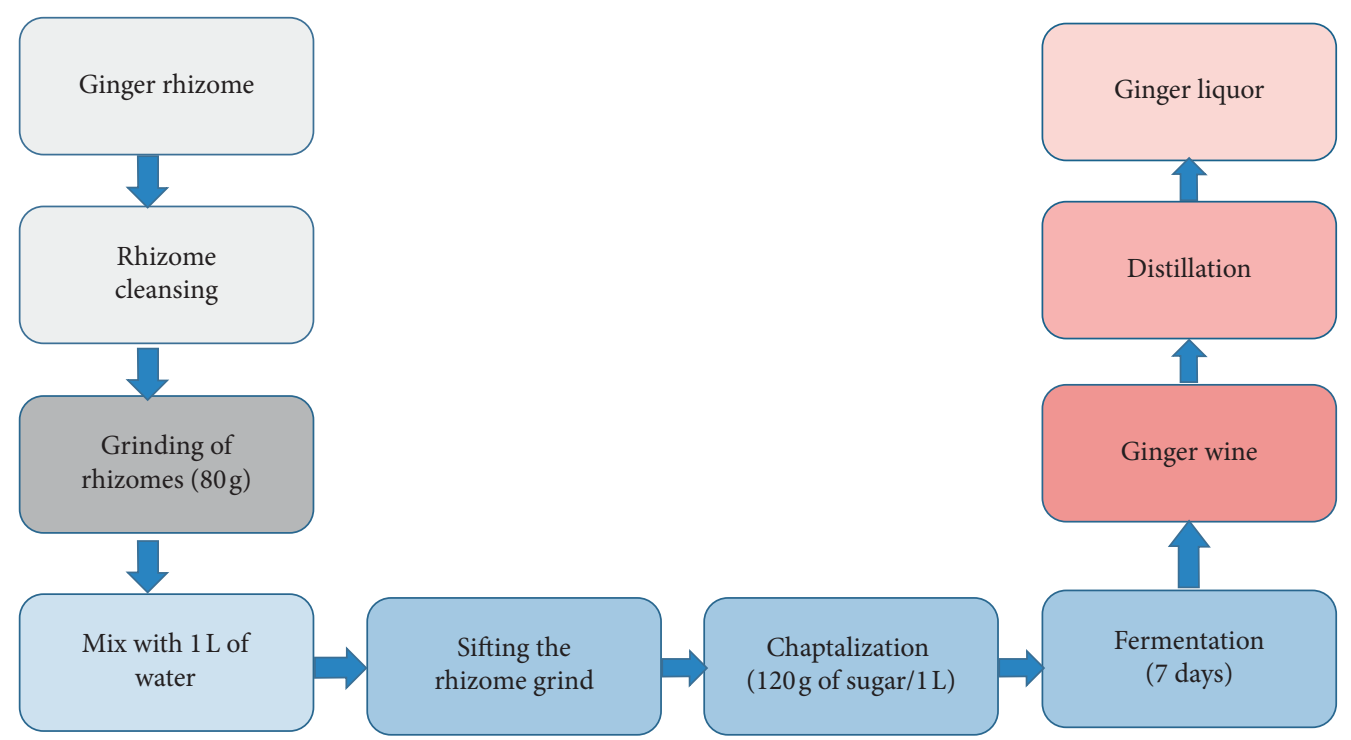

Figure 1: The lab process of fermentation of ginger wine.

the unsweetened sample, the flavonoid concentration varies from 1.39 to $2.22 \mathrm{mg} \mathrm{Eq} \mathrm{Cat/g} \mathrm{Ms} \mathrm{after} 16$ days of fermentation (Figure 3(b)). After 16 days of fermentation, there is an increase in the concentration, i.e., $2.224 \mathrm{mg}$ Eq Cat/g Ms (FIG. 9). For the sweet sample, the flavonoid concentration varies from 1.31 to $2.39 \mathrm{mg}$ Eq Cat/g Ms (Figure 3(b)).
4.4. Enzymatic Activities in the Bioincrease of Antioxidants. The ability isolates suspected like Bacillus to secrete several exocellular enzymes and to coordinate the action with the increase of antioxidants in the fermentation process of ginger juice allow us to find out for enzymatic activities. Isolates have been tested for their ability to degrade milk 


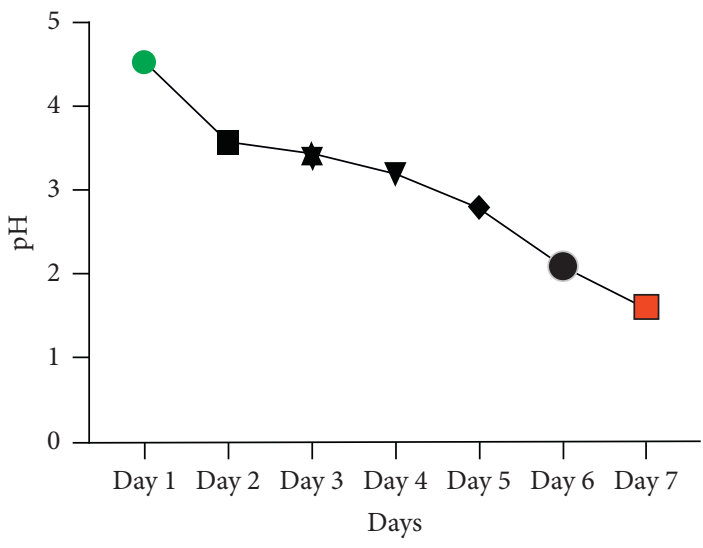

(a)

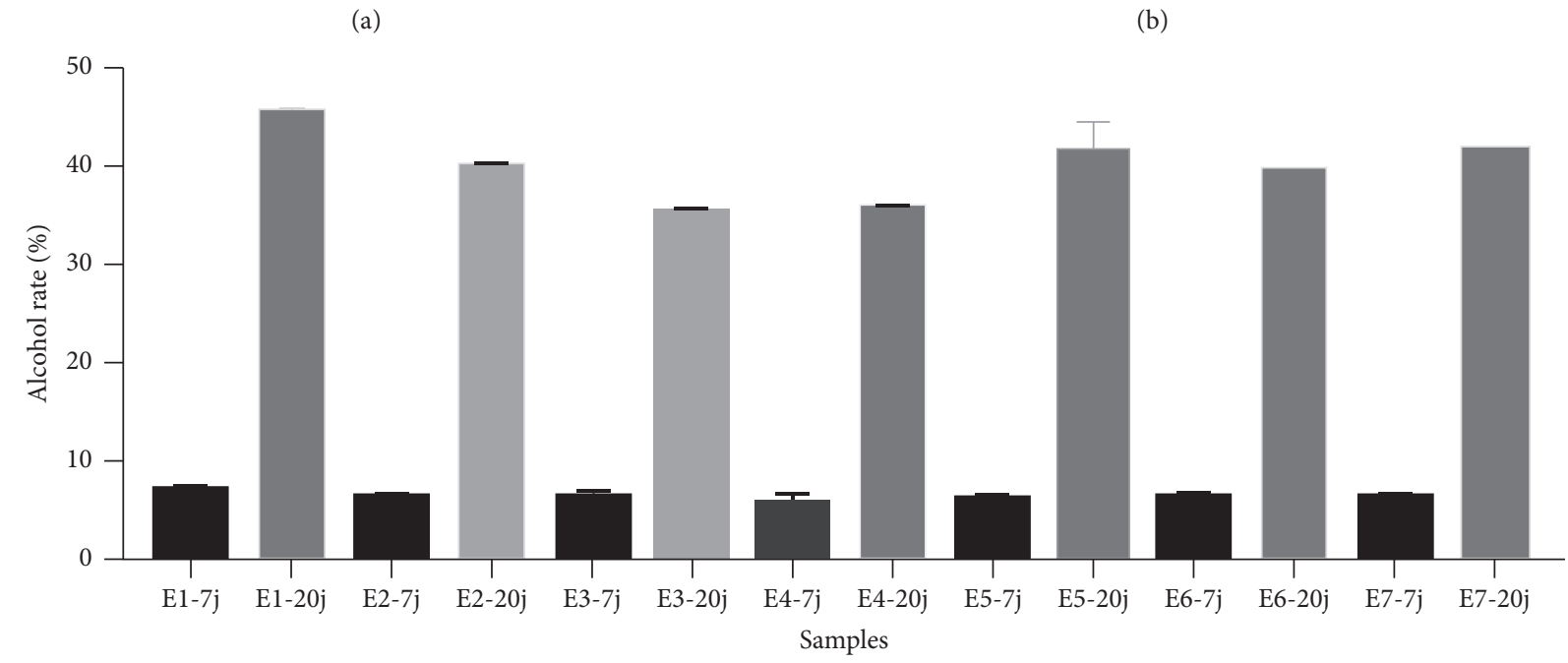

(c)

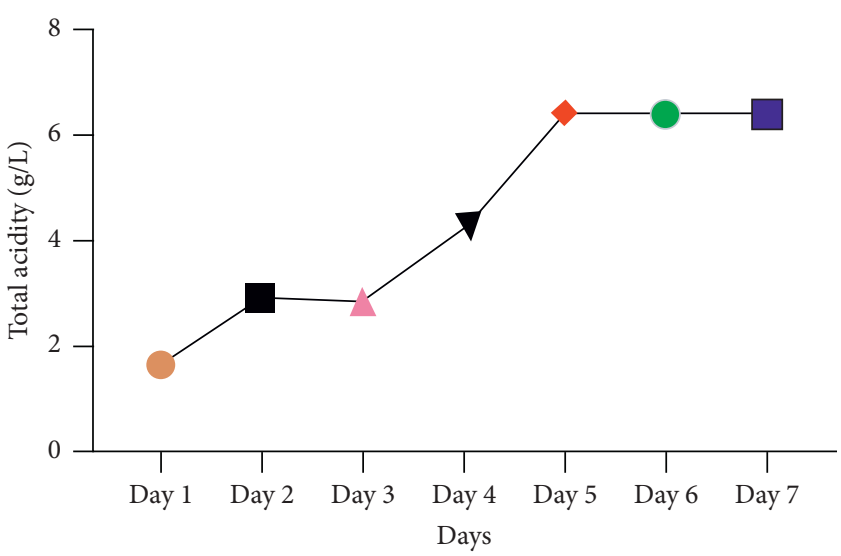

(b) 


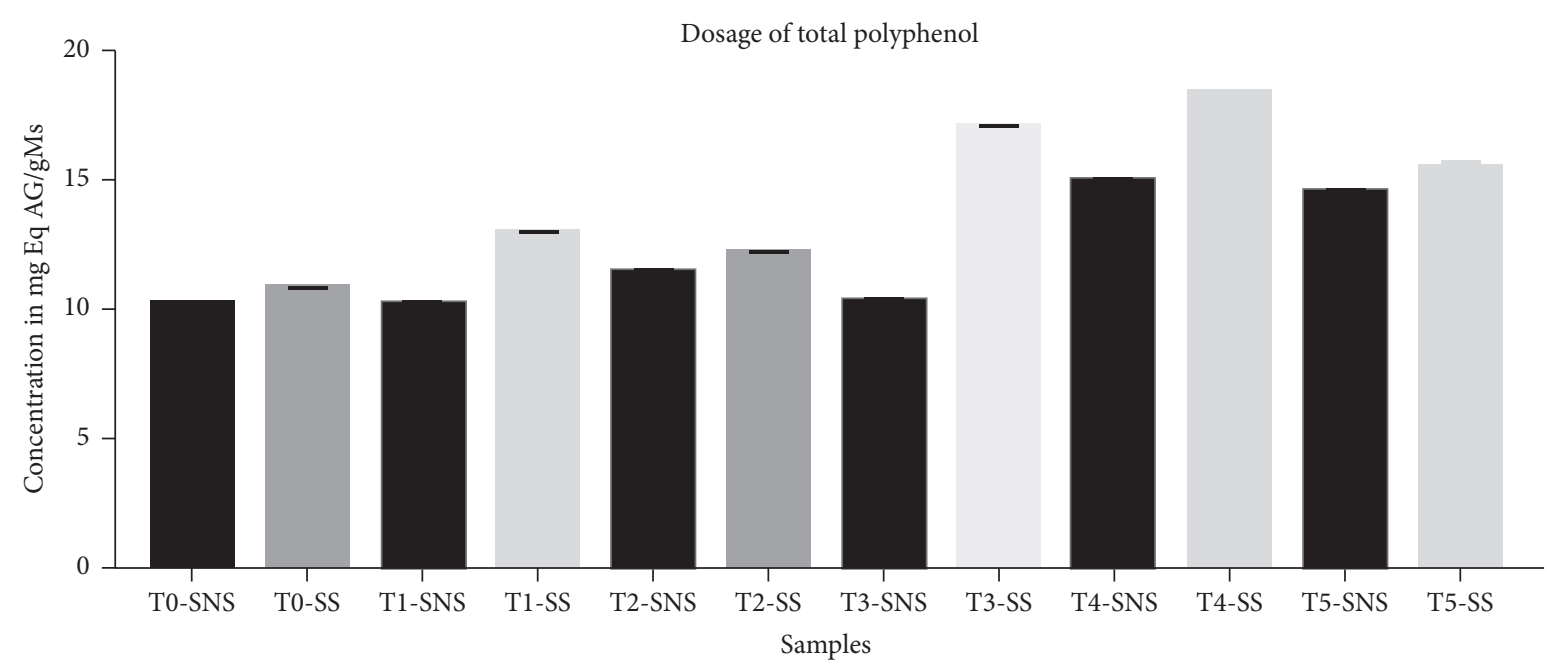

(a)

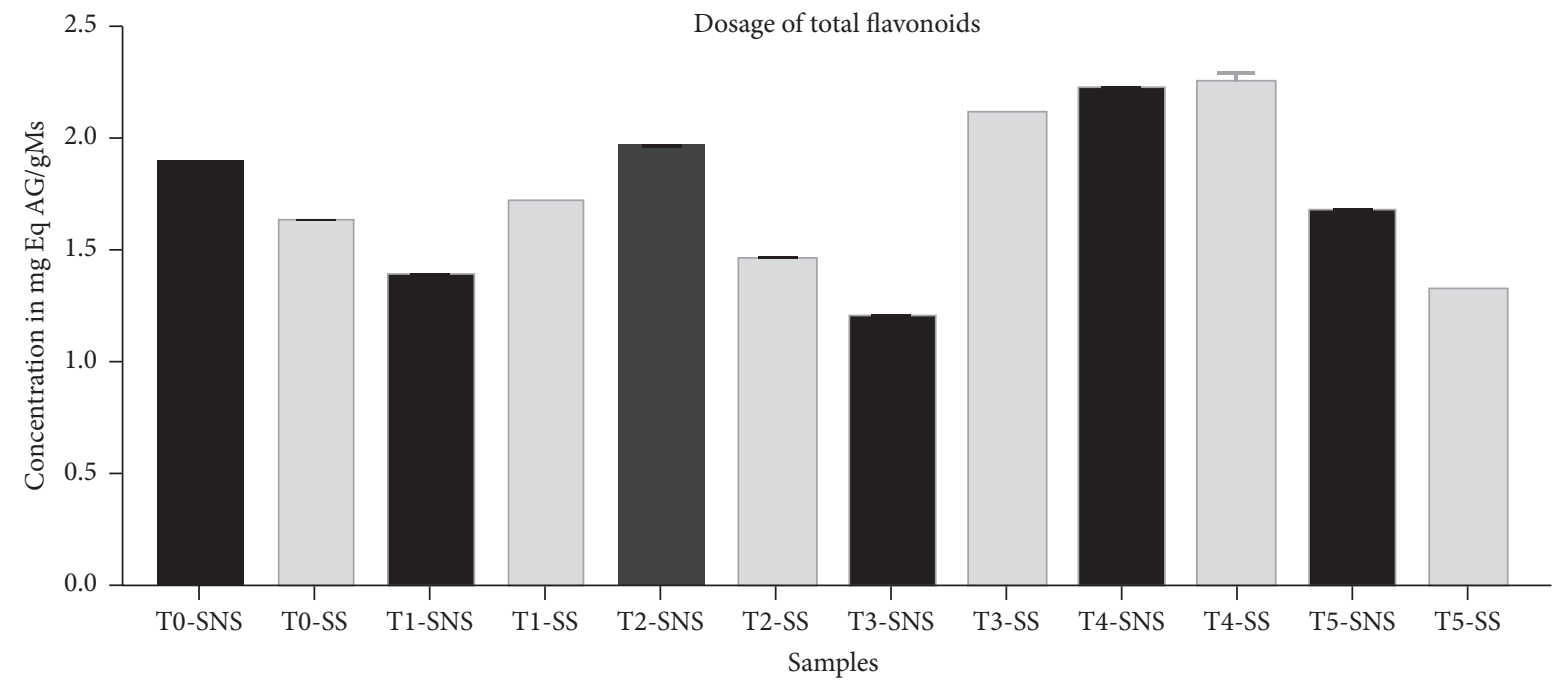

(b)

FIGURE 3: Bioincrease of polyphenol (a) and flavonoids (b) during the fermentation of ginger juice from a sweet sample (SS) and nonsugar added sample (SNS). T0: first day, T1: fourth day after fermentation, T2: eighth day after fermentation, T3: twelfth day after fermentation, T4: sixteenth day after fermentation, and T5: twentieth day after fermentation.

including Saccharomyces cerevisiae, S. mikatae, S. paradoxus, S. arboricolus, S. kudriavzevii, and S. bayanus. Agarose gel electrophoresis revealed a single band at $150 \mathrm{bp}$ corresponding to the isolates OM4, OM5, OM13, OM15, OM19, and OM20. All isolates were assigned to $S$. cerevisiae (Figure 7(a)). Other isolates have not been identified based on the targeted species (Figure 7(a)).

Isolates with good profiles based on enzyme activities were selected for the extraction of genomic DNA. A total of seven isolates were selected: RM1, 3, 5, 7, 12, 19, 23, 40, and 41. The revelation was made on $1 \%$ agarose gel by using BET. After the amplification using the specific primers, the results show that only three pairs of specific primers allowed the amplification of the fibE gene, in particular the couple fibE-Bp, fibE-Bl, and fibE-Bsa, primers targeting, respectively, $B$. pumilus, $B$. licheniformis, and $B$. safensis (Figure 7(b)).

\section{Discussion}

The aim of this work was to understand the involvement of microorganisms in the bioincrease of phenolic compounds during the fermentation of ginger juice. In this way, we have first of all made the ginger wine and, as a result of biochemical analysis, the alcoholic degree after distillation is between 35 and $45 \%$. These high levels of alcohol could be explained by the presence of microorganisms such as yeasts [23] promoting with chaptalization which is an additional advantage of microorganisms. Titratable acidity is a measure of the total acid concentration. In the titration with a base, all the $\mathrm{H}+$ ions are neutralized whether they are ionized or not. We have also shown that titratable acidity increases during fermentation. It turns out that the average acidity ranges between 1.687 and $6.431 \mathrm{~g} / \mathrm{L}$. This acidity is caused by the fermentation activity of microorganisms which degrade cell 


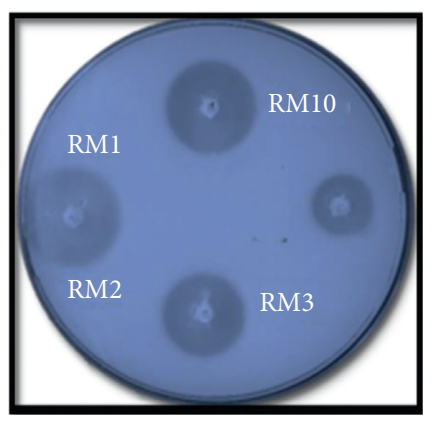

(a)

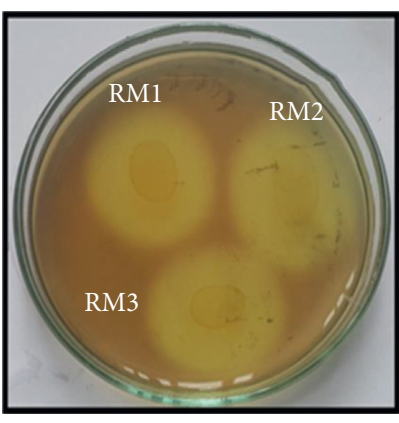

(b)

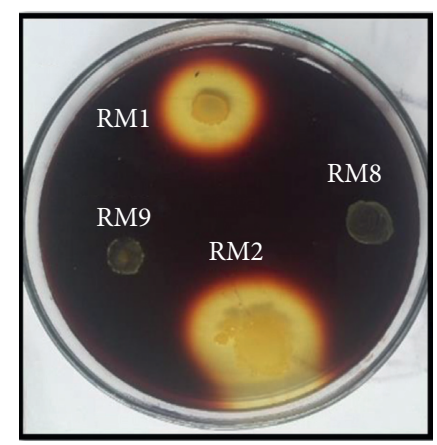

(c)

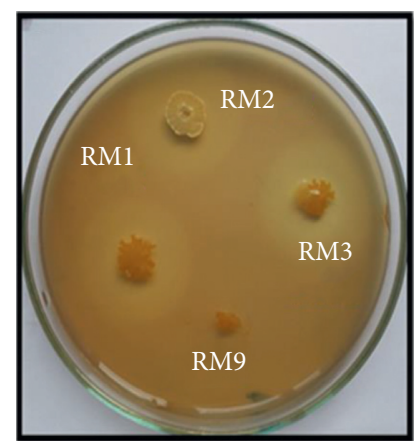

(d)

FIgURe 4: Illustration of some enzymatic activities carried out on isolates from ginger wine. (a) Proteolytic activity. (b) Cellulolytic activity. (c) Amylolytic activity. (d) Pectinolytic activity. RM1, 2, 3, 8, 9, and 10: isolates.

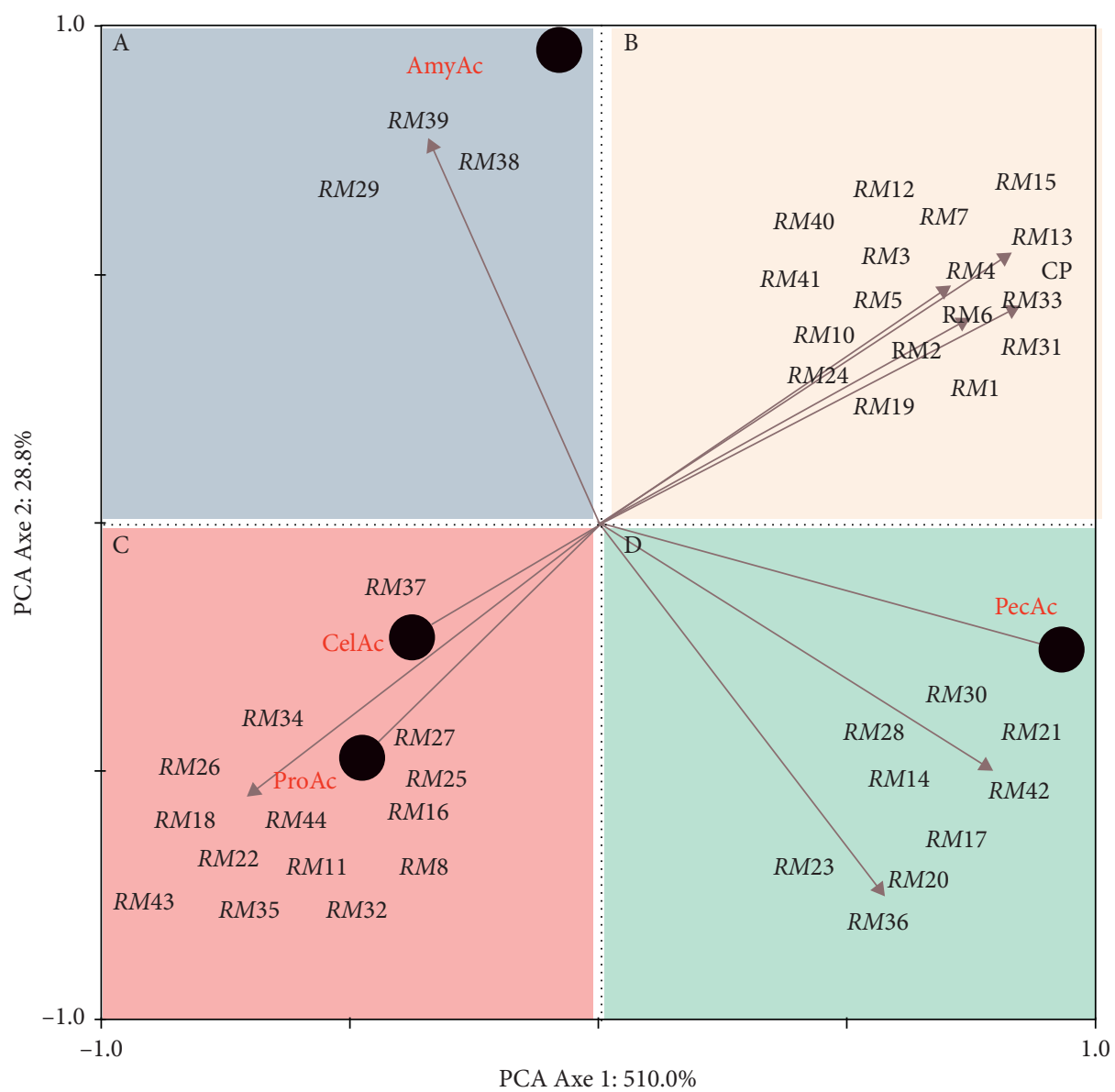

(a)

Figure 5: Continued. 


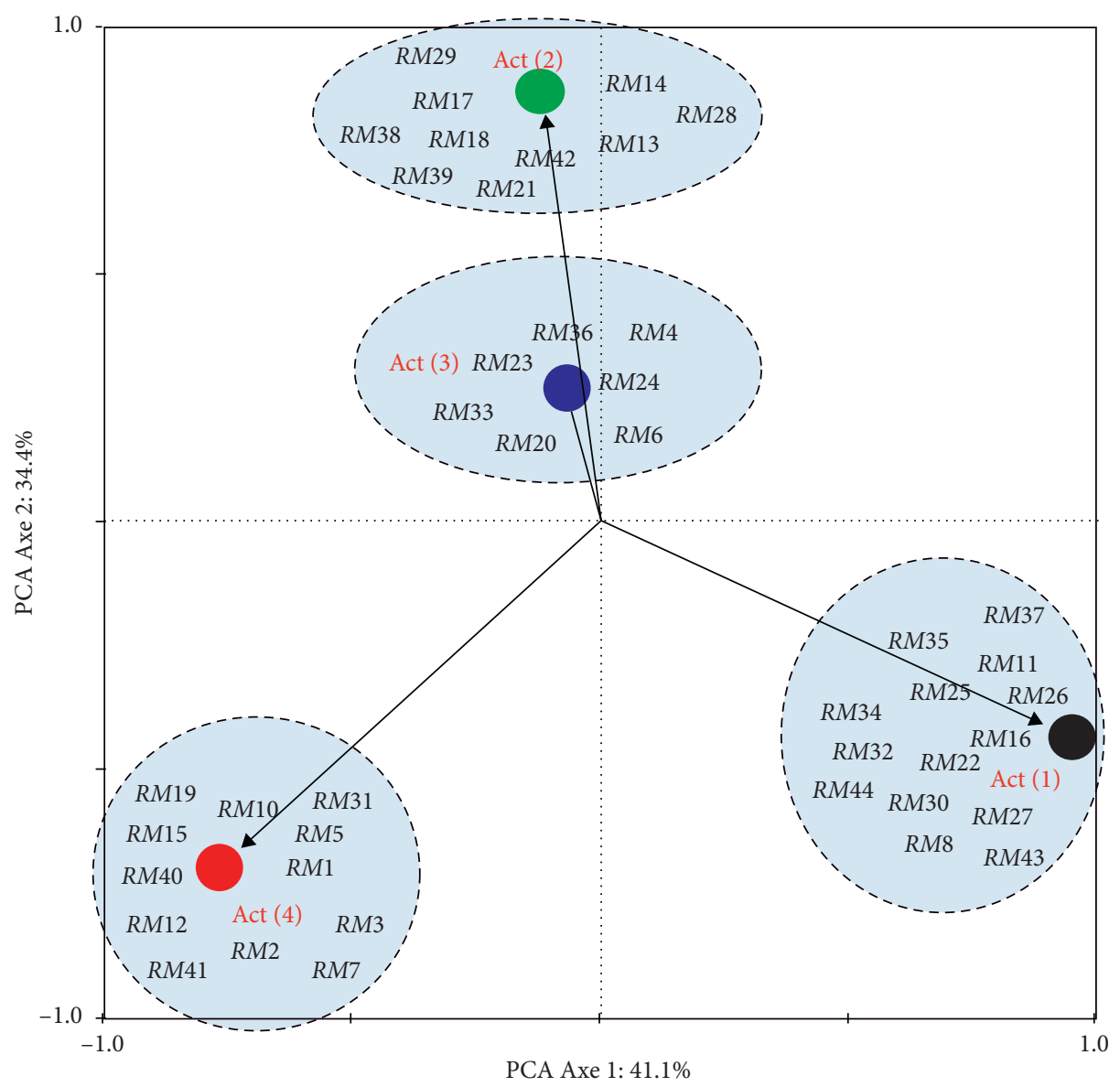

(b)

FIgURe 5: (a) PCA of isolates with one enzymatic activity: proteolytic (ProAc), cellulolytic (CelAc), amylolytic (AmyAc), and pectinolytic (PecAc). (b) PCA of isolates with one enzymatic activity (Act1), 2 enzymatic activities (Act2), 3 enzymatic activities (Act3), and 4 enzymatic activities (Act4): PCA: principal component analysis. RM1 to 44: isolates 1 to 44 .

wall by releasing some organic acids in the medium [24]. The $\mathrm{pH}$ has shown values down to $1.6 \pm 0.1$. Some fermented drinks have been shown with values around $4.5 \pm 0.2$. The type of fermentation assigned to pineapple wine is purely lactic fermentation. Ginger rhizomes are rich in omega 3 and 9 fatty acids (oleic and linolenic acids) [5]. The coordinated action of enzymes secreted by yeasts and bacteria of the Bacillus genus would help acidify ginger wine. The $\mathrm{pH}$ indicates the acidity and the alkalinity of a product while the titratable acidity indicates the quantity of acids present in the ginger juice. The $\mathrm{pH}$ and titratable acidity of the ginger juice were used to estimate the consumability and properties that are not visible. Both criteria can be considered as indicators of organoleptic characteristics. Acids play an important role in the quality of modern or traditional wines because the flavor is essentially and indisputably a balance between the sugar and acid content.

In this work, we have shown that polyphenols and flavonoids increase during fermentation of ginger. Microorganisms are strongly implicated in this process $[14,16,17,25]$. Using molecular biology techniques, we have shown Saccharomyces cerevisiae is mainly isolated from ginger wine as well as bacteria of the genus Bacillus like $B$. licheniformis, B. safensis, and Bacillus pumilus. Together with Saccharomyces cerevisiae, they play a primordial role in the bioincrease of the production of polyphenols and flavonoids [26]. Saccharomyces cerevisiae has been shown to produce $\beta$-glucosidase and feruloyl esterase for cell wall degradation $[18,27]$. The fibE gene encoding a fibrinolytic enzyme was used as a biomarker to identify isolates. This method has an advantage of being reliable and rapid and has great discriminatory power as shown by Kaya-Ongoto et al. [19]. In total, three strains of Bacillus including B. licheniformis, $B$. pumilus, and B. safensis belonging to phylogenic group I were identified. These results are consistent with previous works showing that bacteria of the genus Bacillus can be isolated from fermented foods [19, 28-31].

The total polyphenol concentrations of the unsweetened sample vary from 10.18 to $14.64 \mathrm{mg} \mathrm{Eq} \mathrm{AG/g} \mathrm{Ms.} \mathrm{As} \mathrm{fa} \mathrm{as} \mathrm{the}$ sweet sample is concerned, values vary from 10.82 to $18.34 \mathrm{mg}$ Eq AG/g Ms. The flavonoid concentrations in the unsweetened sample range from 1.394 to $2.224 \mathrm{mg} \mathrm{Eq} \mathrm{Cat/g.}$ The flavonoid concentrations in the sweet sample vary from 1.311 to $2.290 \mathrm{mg} \mathrm{Eq} \mathrm{Cat/g}$. It is possible to accept that chaptalization is an important factor for promoting the growth of yeasts. The high density of microorganisms linked 


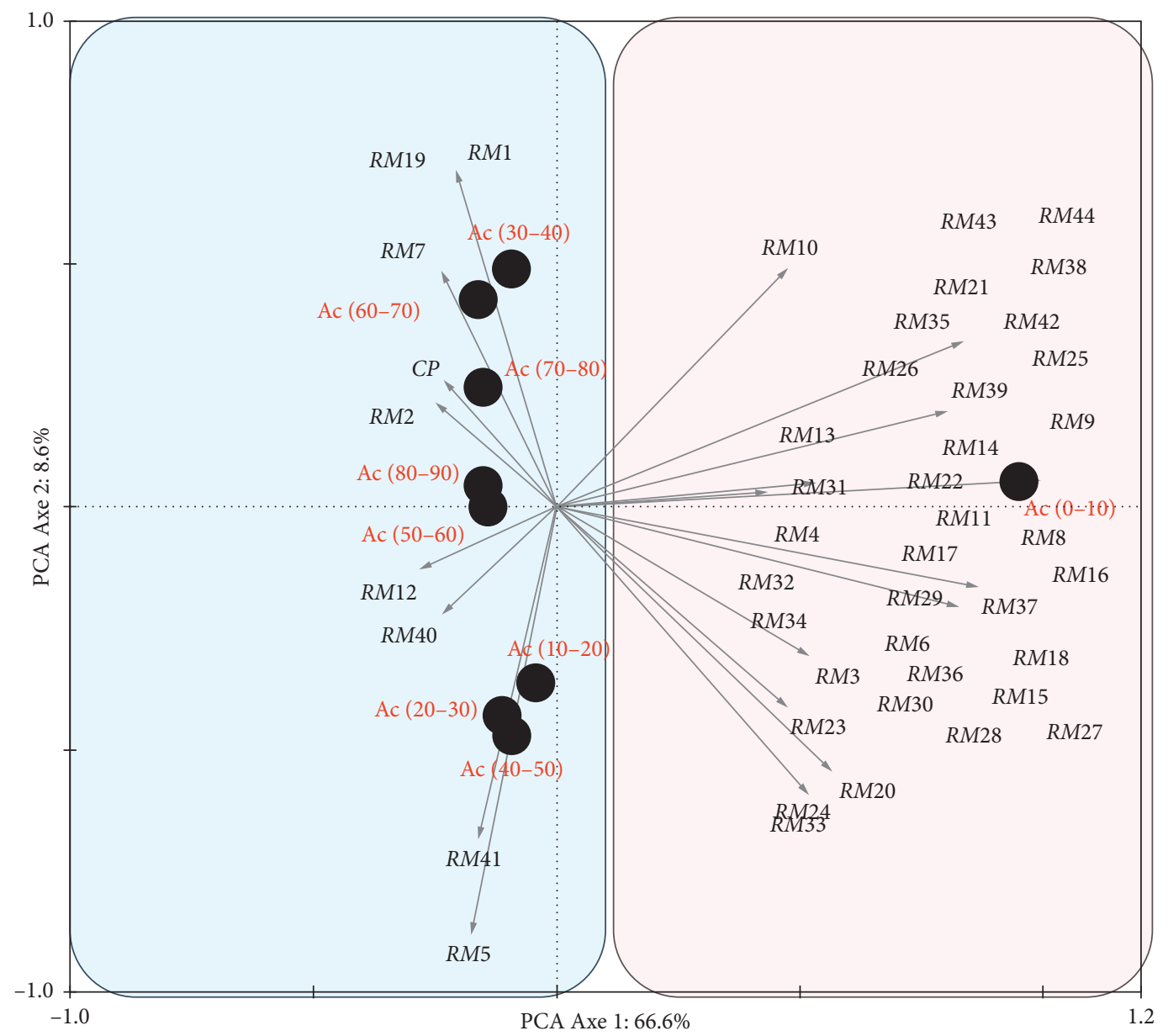

FIgURE 6: PCA of isolates with interesting percentage of enzymatic activities: proteolytic, cellulolytic, amylolytic, and pectinolytic. PCA: principal component analysis. RM1 to 44: isolates 1 to 44 .

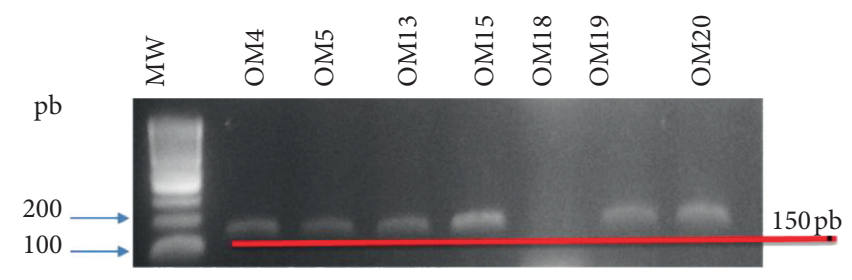

(a)

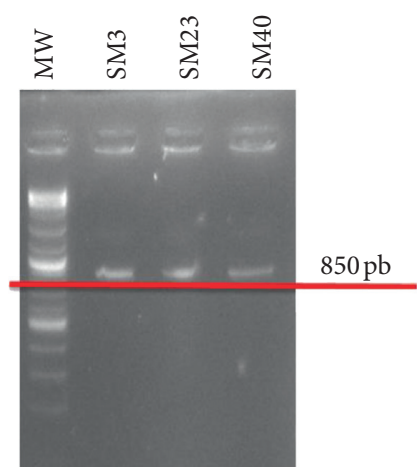

(b)

Figure 7: (a) 1\% agarose gel electrophoresis after amplification using 2 pairs of oligonucleotides of the isolates (OM4, OM5, OM13, OM15, OM17, OM18, OM19, and OM20). MW: molecular weight. (b) The electrophoretic profile on agarose gel (1\%) obtained using the fibEspecific primers to each species of Bacillus: SM3, S23, and SM40.

to the secretion of pectinolytic, amylolytic, and cellulolytic enzymes explains this increase. These enzymes are directly responsible for the destruction of the cell walls of plant cells, thereby causing this increase [26]. However, we note that in the nonchaptalized samples (no added sugar), there is also an increase in total phenolic compound and total flavonoids as well. This increase is explained by the composition of crop that contains sucrose, glucose, and fructose [32, 33] which allow microorganisms to grow and to take a benefit in the environment carbon. Soluble and insoluble carbohydrates 
are the most important components in crop rhizome and contribute to their nutritive value [33]. Given the importance of polyphenols as antioxidants, one could safely say that the fermentation of ginger juice offers the possibility of studying polyphenols and extracting them. This first study needs to be deepened by targeting other types of polyphenols useful to contribute to the metabolic pathways for good health. The decrease in total polyphenols and flavonoids after the seventh day can be explained by the fact that these compounds can also become potential sources of carbon of microorganisms. Interactional synergies of Saccharomyces cerevisiae and Bacillus sp., the emergence of new microorganism groups during fermentation could impact the increase and/or decrease in total polyphenols.

The genus Bacillus is known for its ability to produce extracellular enzymes such as amylases [34], pectinases [35-37], cellulases [18, 38, 39], proteases [40, 41], and other biomolecules as well [42]. In the context of this work, the results are promising because some identified isolates and nonidentified ones have shown degradation percentages of cellulose, amylose, and pectin up to $30 \%$ depending on the activity (cellulolytic, pectinolytic, or amylolytic) and also lysis diameters of the order of $2.2 \mathrm{~cm} \pm 0.03$ in the case of proteolytic activity. All the enzymatic arsenal could thus make it possible to degrade the wall of the plant cell by allowing the release of biomolecules like the antioxidants, being inside the plant cells of the ginger rhizomes towards the outside. The enzymatic activities carried out have shown that bacteria of the genus Bacillus secrete lytic enzymes produced during fermentation that could contribute to the degradation of cell wall structures resulting in biomolecular release such as polyphenols and flavonoids. This therefore optimizes the amount of polyphenols in this drink [43]. Patients suffering from diabetes mellitus [44] and obesity $[45,46]$ are not obliged to chaptalize ginger juice in order to have a benefit from the polyphenols released during the fermentation orchestrated by microorganisms. In this work, we have shown that nonchaptalized ginger juice can also be bioincreased. Indeed, microorganisms like Saccharomyces sp. and Bacillus sp. isolated from ginger wine are also able to use the sugars of rhizomes and many other molecules [5] by releasing the enzymes which will destroy the walls of plant cells.

Several studies reported that fermentation influences the polyphenolic profile of extracts obtained from various plant sources or during the fermentation of plant sources. This concerns Bacillus pumilus and Bacillus subtilis on the fermentation of soybean and cheonggukjang [16, 47]; Lactobacillus acidophilus on apple juice; Lactobacillus plantarum, Lactobacillus acidophilus, Lactobacillus johnsonii, Lactobacillus reuteri, Lactobacillus acidophilus, and Lactobacillus delbrueckii on whole grain barley, oat groat, and soybean $[14,48]$; and Saccharomyces cerevisiae on wheat bran [17]. In this work, we demonstrate that Saccharomyces cerevisiae, Bacillus licheniformis, Bacillus pumilus, and Bacillus safensis can easily and synergically play this role by using both genera enzymatic landscapes that can degrade plant material.

\section{Conclusion}

The consumption of rhizomes or ginger juice is a real health ally. The nutritional quality of people with diabetes remains a real challenge in the Republic of the Congo. In this work, we have shown that the fermentation process of ginger juice is necessary to obtain the high values of antioxidants like polyphenols and flavonoids. People with diabetes and obesity can also consume ginger without the need to add sugar. Microorganisms like Saccharomyces cerevisiae, Bacillus pumilus, Bacillus licheniformis, and Bacillus safensis secrete enzymes which are able to bioincrease polyphenols and especially flavonoids during the fermentation of ginger juice. More studies are on the way to optimize other bioactive substances and/or fermentation conditions in Congo ginger wine.

\section{Data Availability}

The Excel sheet including the data used to support the findings of this study is available from the corresponding author upon request.

\section{Conflicts of Interest}

The authors declare no conflicts of interest.

\section{Acknowledgments}

The authors are grateful to Dr. Aimé Bertrand Madiélé Mabika and Dr. Tsiba Gouollally (Faculté des Sciences et Techniques, Université Marien Ngouabi) for the technical support and to Prof. Clobite Bouka Biona (Institut National de Recherche en Sciences Exactes et Naturelles (IRSEN), Brazzaville, Congo), Prof. Arsen Lenga (Faculté des Sciences et Techniques, Université Marien N'GOUABI), and Prof Michel Dzondo Gadet (Ecole Normle Supérieure Polytechnique, Université Marien N'GOUABI) for their continuous encouragement and helpful data analysis before publication.

\section{References}

[1] Y. A. Han, C. W. Song, W. S. Koh et al., "Anti-inflammatory effects of the Zingiber officinale roscoe constituent 12-dehydrogingerdione in lipopolysaccharide-stimulated raw 264.7 cells," Phytotherapy Research, vol. 27, no. 8, pp. 1200-1205, 2013.

[2] S. Chrubasik, M. H. Pittler, and B. D. Roufogalis, "Zingiberis rhizoma: a comprehensive review on the ginger effect and efficacy profiles," Phytomedicine, vol. 12, no. 9, pp. 684-701, 2005.

[3] R. Haniadka, E. Saldanha, V. Sunita, P. L. Palatty, R. Fayad, and M. S. Baliga, "A review of the gastroprotective effects of ginger (Zingiber officinale Roscoe)," Food and Function, vol. 4, no. 6, pp. 845-855, 2013.

[4] S. Suk, G. T. Kwon, E. Lee et al., "Gingerenone A, a polyphenol present in ginger, suppresses obesity and adipose tissue inflammation in high-fat diet-fed mice," Molecular Nutrition \& Food Research, vol. 61, no. 10, Article ID 1700139, 2017. 
[5] Q.-Q. Mao, X.-Y. Xu, S.-Y. Cao et al., "Bioactive compounds and bioactivities of ginger (Zingiber officinale roscoe)," Foods, vol. 8, no. 6, p. 185, 2019.

[6] K. Ji, L. Fang, H. Zhao et al., "Ginger oleoresin alleviated gamma-ray irradiation-induced reactive oxygen species via the Nrf2 protective response in human mesenchymal stem cells," Oxidative Medicine and Cellular Longevity, vol. 2017, Article ID 1480294, 12 pages, 2017.

[7] A. O. Abolaji, M. Ojo, T. T. Afolabi, M. D. Arowoogun, D. Nwawolor, and E. O. Farombi, "Protective properties of 6gingerol-rich fraction from Zingiber officinale (Ginger) on chlorpyrifos-induced oxidative damage and inflammation in the brain, ovary and uterus of rats," Chemico-Biological Interactions, vol. 270, pp. 15-23, 2017.

[8] Y.-S. Lai, W.-C. Lee, Y.-E. Lin et al., "Ginger essential oil ameliorates hepatic injury and lipid accumulation in high fat diet-induced nonalcoholic fatty liver disease," Journal of Agricultural and Food Chemistry, vol. 64, no. 10, pp. 20622071, 2016.

[9] Y. Kawamoto, Y. Ueno, E. Nakahashi et al., "Prevention of allergic rhinitis by ginger and the molecular basis of immunosuppression by 6-gingerol through T cell inactivation," The Journal of Nutritional Biochemistry, vol. 27, pp. 112-122, 2016.

[10] C. Sampath, M. R. Rashid, S. Sang, and M. Ahmedna, "Specific bioactive compounds in ginger and apple alleviate hyperglycemia in mice with high fat diet-induced obesity via Nrf2 mediated pathway," Food Chemistry, vol. 226, pp. 79-88, 2017.

[11] M. Pinelo, A. Arnous, and A. S. Meyer, "Upgrading of grape skins: significance of plant cell-wall structural components and extraction techniques for phenol release," Trends in Food and Sciences Technology, vol. 17, no. 11, pp. 579-590, 2006.

[12] E. Jablonska-Rys, A. Slawinska, and D. Szwajgier, "Effect of lactic acid fermentation on antioxidant properties and phenolic acid contents of oyster (Pleurotus ostreatus) and chanterelle (Cantharellus cibarius) mushrooms," Food Science and Biotechnology, vol. 25, no. 2, pp. 439-444, 2016.

[13] R. D. Cagno, G. Minervini, C. G. Rizzello, M. D. Angelis, and M. Gobbetti, "Effect of lactic acid fermentation on antioxidant, texture, color and sensory properties of red and green smoothies," Food Microbiology, vol. 28, no. 5, pp. 1062-1071, 2011.

[14] Y.-H. Pyo, T.-C. Lee, and Y.-C. Lee, "Effect of lactic acid fermentation on enrichment of antioxidant properties and bioactive isoflavones in soybean," Journal of Food Science, vol. 70, no. 3, pp. S215-S220, 2005.

[15] D. Hunaefi, D. N. Akumo, H. Riedel, and I. Smetanska, "The effect of Lactobacillus plantarum ATCC 8014 and Lactobacillus acidophilus NCFM fermentation on antioxidant properties of selected in vitro sprout culture of orthosiphon aristatus (java tea) as a model study," Antioxidants, vol. 1, no. 1, pp. 4-32, 2012.

[16] I.-M. Chung, S.-H. Seo, J.-K. Ahn, and S.-H. Kim, "Effect of processing, fermentation, and aging treatment to content and profile of phenolic compounds in soybean seed, soy curd and soy paste," Food Chemistry, vol. 127, no. 3, pp. 960-967, 2011.

[17] J. Moore, Z. Cheng, J. Hao et al., "Effects of solid-state yeast treatment on the antioxidant properties and protein and fiber compositions of common hard wheat bran," Journal of Agricultural and Food Chemistry, vol. 55, no. 25, pp. 1017310182, 2007.

[18] S. J. Hur, S. Y. Lee, Y.-C. Kim, I. Choi, and G.-B. Kim, "Effect of fermentation on the antioxidant activity in plant-based foods," Food Chemistry, vol. 160, pp. 346-356, 2014.
[19] M. D. Kaya-Ongoto, C. A. Kayath, E. Nguimbi et al., "Genetic clearness novel strategy of group I Bacillus species isolated from fermented food and beverages by using fibrinolytic enzyme gene encoding a serine-like enzyme," Journal of Nucleic Acids, vol. 2019, Article ID 5484896, 13 pages, 2019.

[20] A. Muir, E. Harrison, and A. Wheals, "A multiplex set of species-specific primers for rapid identification of members of the genus Saccharomyces," FEMS Yeast Research, vol. 11, no. 7, pp. 552-563, 2011.

[21] J. R. Fischer and C. P. Paukert, "Habitat relationships with fish assemblages in minimally disturbed great plains regions," Ecology of Freshwater Fish, vol. 17, no. 4, pp. 597-609, 2008.

[22] C. J. F. T. Braak and P. Šmilauer, Canoco 4, Cambridge University Press, New York, NY, USA, 2003.

[23] M. S. Silva, L. M. Arruda, P. L. Xavier et al., "Selection of yeasts from bee products for alcoholic beverage production," Brazilian Journal of Microbiology, vol. 51, no. 1, pp. 323-334, 2019.

[24] S. A. Villarreal-Soto, S. Beaufort, J. Bouajila, J.-P. Souchard, and P. Taillandier, "Understanding kombucha tea fermentation: a review," Journal of Food Science, vol. 83, no. 3, pp. 580-588, 2018.

[25] J.-H. Ye, L.-Y. Huang, N. S. Terefe, and M. A. Augustin, "Fermentation-based biotransformation of glucosinolates, phenolics and sugars in retorted broccoli puree by lactic acid bacteria," Food Chemistry, vol. 286, pp. 616-623, 2019.

[26] K. M. Cho, S. Y. Hong, R. K. Math et al., "Biotransformation of phenolics (isoflavones, flavanols and phenolic acids) during the fermentation of cheonggukjang by Bacillus pumilus HY1," Food Chemistry, vol. 114, no. 2, pp. 413-419, 2009.

[27] S. Coghe, K. Benoot, F. Delvaux, B. Vanderhaegen, and F. R. Delvaux, "Ferulic acid release and 4-vinylguaiacol formation during brewing and fermentation: indications for feruloyl esterase activity in Saccharomyces cerevisiae," Journal of Agricultural and Food Chemistry, vol. 52, no. 3, pp. 602608, 2004.

[28] L. I. I. Ouoba, B. Diawara, W. K. Amoa-Awua, A. S. Traore, and P. L. Moller, "Genotyping of starter cultures of Bacillus subtilis and Bacillus pumilus for fermentation of African locust bean (Parkia biglobosa) to produce Soumbala," International Journal of Food Microbiology, vol. 90, no. 2, pp. 197-205, 2004.

[29] L. I. I. Ouoba, B. Diawara, L. Jespersen, and M. Jakobsen, "Antimicrobial activity of Bacillus subtilis and Bacillus pumilus during the fermentation of African locust bean (Parkia biglobosa) for Soumbala production," Journal of Applied Microbiology, vol. 102, no. 4, pp. 963-970, 2007.

[30] L. I. Ouoba, C. Parkouda, B. Diawara, C. Scotti, and A. H. Varnam, "Identification of Bacillus spp. from Bikalga, fermented seeds of Hibiscus sabdariffa: phenotypic and genotypic characterization," Journal of Applied Microbiology, vol. 104, no. 1, pp. 122-131, 2008.

[31] L. I. Ouoba, K. B. Rechinger, V. Barkholt, B. Diawara, A. S. Traore, and M. Jakobsen, "Degradation of proteins during the fermentation of African locust bean (Parkia biglobosa) by strains of Bacillus subtilis and Bacillus pumilus for production of Soumbala," Journal of Applied Microbiology, vol. 94, no. 3, pp. 396-402, 2003.

[32] K. U. Pradeep, P. Geervani, and B. O. Eggum, "Common Indian spices: nutrient composition, consumption and contribution to dietary value," Plant Foods for Human Nutrition, vol. 44, no. 2, pp. 137-148, 1993.

[33] A. Ghasemzadeh, H. Jaafar, E. Karimi, and S. Ashkani, "Changes in nutritional metabolites of young ginger (Zingiber 
officinale roscoe) in response to elevated carbon dioxide," Molecules, vol. 19, no. 10, pp. 16693-16706, 2014.

[34] V. Singh, R. Sharma, and P. Sharma, "Isolation, screening and optimization of amylase producing Bacillus sp. from soil," Asian Pacific Journal of Health Sciences, vol. 2, no. 3, pp. 86-93, 2015.

[35] P. Yu and C. Xu, "Production optimization, purification and characterization of a heat-tolerant acidic pectinase from Bacillus sp. ZJ1407," International Journal of Biological Macromolecules, vol. 108, pp. 972-980, 2018.

[36] R. B. Mahto, M. Yadav, S. Sasmal, and B. Bhunia, "Optimization of process parameters for production of pectinase using Bacillus subtilis MF447840.1," Recent Patents on Biotechnology, vol. 13, no. 1, pp. 69-73, 2019.

[37] A. Mohandas, S. Raveendran, B. Parameswaran et al., "Production of pectinase from Bacillus sonorensis MPTD1," Food Technology and Biotechnolog, vol. 56, no. 1, pp. 110-116, 2018.

[38] A. Kundu and B. Majumdar, "Optimization of the cellulase free xylanase production by immobilized Bacillus pumilus," Iranian Journal of Biotechnology, vol. 16, no. 4, pp. 273-278, 2018.

[39] L. Sun, J. Cao, Y. Liu, J. Wang, P. Guo, and Z. Wang, "Gene cloning and expression of cellulase of Bacillus amyloliquefaciens isolated from the cecum of goose," Animal Biotechnology, vol. 28, no. 1, pp. 74-82, 2017.

[40] Z. Yao, J. A. Kim, and J. H. Kim, "Characterization of a fibrinolytic enzyme secreted by Bacillus velezensis BS2 isolated from sea squirt jeotgal," Journal of Microbiology and Biotechnology, vol. 29, no. 3, pp. 347-356, 2019.

[41] K. Heo, K. M. Cho, C. K. Lee et al., "Characterization of a fibrinolytic enzyme secreted by Bacillus amyloliquefaciens CB1 and its gene cloning," Journal of Microbiology and Biotechnology, vol. 23, no. 7, pp. 974-983, 2013.

[42] R. Radhakrishnan, A. Hashem, and E. F. Abd-Allah, "Bacillus: a biological tool for crop improvement through bio-molecular changes in adverse environments," Frontiers in Physiology, vol. 8, p. 667, 2017.

[43] N. T. Huynh, J. V. Camp, G. Smagghe, and K. Raes, "Improved release and metabolism of flavonoids by steered fermentation processes: a review," International Journal of Molecular Sciences, vol. 15, no. 11, pp. 19369-19388, 2014.

[44] Y. Zhu, Y. Zhao, P. Wang, M. Ahmedna, and S. Sang, "Bioactive ginger constituents alleviate protein glycation by trapping methylglyoxal," Chemical Research in Toxicology, vol. 28 , no. 9, pp. 1842-1849, 2015.

[45] K. Misawa, K. Hashizume, M. Yamamoto, Y. Minegishi, T. Hase, and A. Shimotoyodome, "Ginger extract prevents high-fat diet-induced obesity in mice via activation of the peroxisome proliferator-activated receptor delta pathway," The Journal of Nutritional Biochemistry, vol. 26, no. 10, pp. 1058-1067, 2015.

[46] R. H. Mahmoud and W. A. Elnour, "Comparative evaluation of the efficacy of ginger and orlistat on obesity management, pancreatic lipase and liver peroxisomal catalase enzyme in male albino rats," European Review for Medical and Pharmacological Sciences, vol. 17, no. 1, pp. 75-83, 2013.

[47] A. Ghasemzadeh, H. Z. E. Jaafar, and A. Rahmat, "Changes in antioxidant and antibacterial activities as well as phytochemical constituents associated with ginger storage and polyphenol oxidase activity," BMC Complementary and Alternative Medicine, vol. 16, no. 1, p. 382, 2016.

[48] A. S. Hole, I. Rud, S. Grimmer, S. Sigl, J. Narvhus, and S. Sahlstrom, "Improved bioavailability of dietary phenolic acids in whole grain barley and oat groat following fermentation with probiotic Lactobacillus acidophilus, Lactobacillus johnsonii, and Lactobacillus reuteri," Journal of Agricultural and Food Chemistry, vol. 60, no. 25, pp. 63696375, 2012. 